\title{
Optimal Quantization in Decentralized Detection by Maximizing the Average Entropy of the Sensors
}

\author{
Muath A. Wahdan and Mustafa A. Altınkaya \\ Department of Electrical and Electronics Engineering \\ İzmir Institute of Technology \\ İzmir, Turkey \\ muathwahdan@iyte.edu.tr and mustafaaltinkaya@iyte.edu.tr
}

\begin{abstract}
In a wireless sensor network the sensor outputs are required to be quantized because of energy and bandwidth requirements. We propose such a distributed detection scheme for a point source which is based on Neyman-Pearson criterion where sensor outputs are quantized maximizing the average output entropy of the sensors under both hypotheses. The quantized local outputs are transmitted to a fusion center (FC) where they are used to make a global decision. The performance of the proposed maximum average entropy (MAE) method in quantizing sensor outputs was tested for binary, ternary and quarternary quantization. The effects of the channel from the sensors to the FC is also addressed by simplified channel models. The simulation studies show the success of the MAE method.

Index Terms-Decentralized detection, quantization, point source, field source, wireless sensor networks (WSNs).
\end{abstract}

\section{INTRODUCTION}

Decentralized detection using sensor networks has received considerable attention in the last decade [1], [2]. Owing to strict energy and bandwidth restrictions, observations of the sensors are frequently needed to be quantized, before transmitting them to a fusion center (FC) where a global decision is made [3]. Distributed detection systems [4] show the advantages of higher survivability and reliability than their centralized counterparts. In [5], the authors consider a detection problem consisting of two sensors and one FC with a fixed fusion rule to show that the optimum local decision rule is the likelihood ratio test under the Bayesian criterion. Then, in [6] and [7], it was shown that the optimum fusion rule at the FC is also a likelihood ratio test both under the Neyman-Pearson and the Bayesian criteria.

Optimum quantization levels in the sense of information theoretic criteria for distributed detection systems were presented in [8], [9]. In [8], J-divergence has been used to optimize the distributed detection of a serial system with two sensors for the Bayesian detection criterion. In [9], optimum quantization levels have been investigated for a binary system under the assumption that the decision thresholds and the decision statistics are given and fixed. Then, they impose additional partitioning of decision regions on the local sensors instead of sending hard decisions in order to provide more detailed information to the FC. In that work, it was assumed that all local sensors are identical NP detectors observing the same signal-to-noise ratio (SNR). In [10], the optimal quantization intervals based on deflection criterion (DC) and Chernoff information (CI) are defined for distributed detection systems consisting of one FC and multiple sensors by using Bayesian detection criterion for known SNR.

In this paper, we propose an entropy based method for determining the quantization intervals at distributed sensors in order to optimize the global binary decision at the fusion center about the existence of a point source under the NeymanPearson criterion where sensors observe different signal levels which they do not know.

This paper is organized as follows. First, the problem statement is given in Section II. Section III covers application of the proposed method for binary, ternary and quarternary quantization of sensor observations for detecting a point source using a wireless sensor network (WSN). The simulation results are given in Section IV. Finally, conclusions are drawn in Section V.

\section{PROBLEM STATEMENT}

A binary hypothesis testing problem has been considered in this paper, where a group of $K$ sensors cooperate to detect the existence of a point source described by the parameter $\mu_{k}$. The problem at each sensor node is given as follows

$$
\begin{gathered}
H_{0}: y_{k}=\epsilon_{k} \\
\text { versus } \\
H_{1}: y_{k}=\mu_{k}+\epsilon_{k},
\end{gathered}
$$

where $y_{k} \in \mathbb{R}$ denotes the $k$ 'th sensor observation as a real number, $\epsilon_{k} \in \mathbb{R}$ denotes additive white Gaussian noise (AWGN) with variance $\sigma^{2}$ and zero mean. $\mu_{k}$ denotes the received signal amplitude from the $k^{\prime}$ th sensor where $k=$ $1,2, \ldots, K$. In this paper, in order to concentrate on the fusion of sensor data with non-identical signal levels, mainly, we assume that error-free sensor outputs are available at the fusion center. We call this as direct data transmission (DDT). However, we have also considered simplified practical channel models to account for the errors introduced at the transmission from the sensors to the FC. Since relative reliability of sensor outputs are not evaluated, equal gain fusion rule is applied.

Based on the dispersion pattern over the surveillance zone and the physical characteristics, the phenomenon to be detected can be modeled either as a field source or a point 


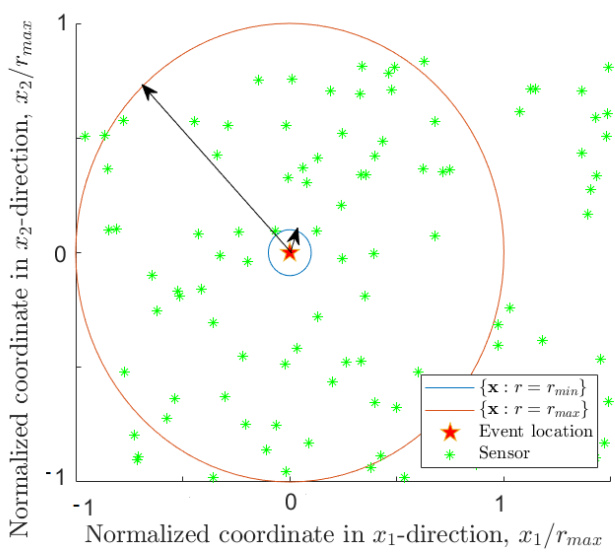

Fig. 1: Positions of the event location and uniformly distributed sensors transmitting to the FC.

source. A field source is dispersed over the sensor field such as in temperature monitoring. On the other hand, the event is generated by a single point source such as in target detection and fire detection. In this work, we consider a point event source emitting constant power uniformly in all directions. For such a source the signal amplitude received by a sensor will be inversely proportional to the distance from the source. Considering uniformly deployed sensors, only those sensors which are within a circle radius, which is determined by the sensitivity of the sensors, will receive a signal.

Let $\mu_{\max }$ denote the signal amplitude on a circle with radius $r_{\text {min }}$ centered by the event location as shown in Figure 1. We assume that $\mu_{\max }$ corresponds to the maximum detectable signal level or the saturation level of the sensors and $\mu_{\min }$ denotes the minimum value of the detectable signal. This yields a different and unknown amplitude value at each individual sensor with known pdf given as:

$$
p\left(\mu_{n}\right)=\frac{1}{\mu_{n} \log (L)}
$$

where $L=\mu_{\max } / \mu_{\min }$ and $\log (\cdot)$ is the natural logarithm. We define the SNR as the ratio between the maximum signal power, $\mu_{\max }^{2}$, and the noise power, $\sigma^{2}$. We use the optimal Bayesian NP detector in our problem which is defined as follows [11]:

$$
\begin{aligned}
& \frac{p\left(\mathbf{y} \mid H_{1}\right)}{p\left(\mathbf{y} \mid H_{0}\right)}=\frac{\int_{\frac{1}{L}}^{1} p\left(\mathbf{y} \mid H_{1} ; \mu_{n}\right) p\left(\mu_{n}\right) d \mu_{n}}{p\left(\mathbf{y} \mid H_{0}\right)} \underset{H_{0}}{\stackrel{H_{1}}{\gtrless} \eta} \\
& \frac{p\left(\mathbf{y} \mid H_{1}\right)}{p\left(\mathbf{y} \mid H_{0}\right)}=\frac{1}{\log (L)} \int_{\frac{1}{L}}^{1} \frac{\exp \left(\frac{-K \mu_{n}^{2}+2 \mu_{n} \sum_{k=1}^{K}\left(y_{k}\right)^{2}}{2 \sigma^{2}}\right)}{\mu_{n}} d \mu_{n} \stackrel{H_{H_{0}}}{\gtrless} \eta
\end{aligned}
$$

where $\mathbf{y}=\left[y_{1}, y_{2}, \ldots, y_{K}\right]$ denotes vector of observations from $K$ sensors.

\section{QUANTIZER DESIGN}

It is aimed to make a global decision at the FC under the NP criterion. Let us assume that each sensor will only

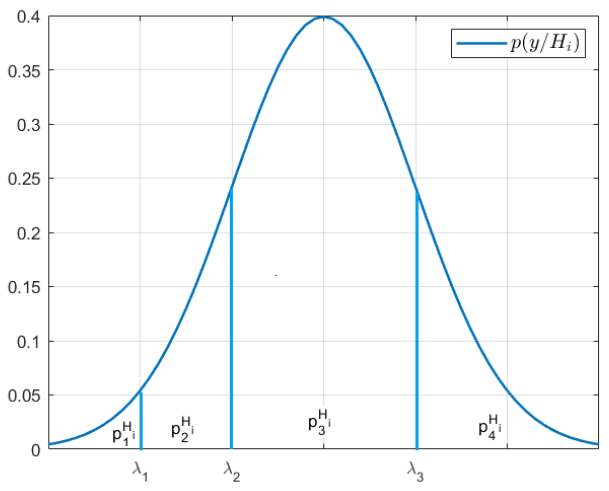

Fig. 2: Partitioning of the pdf for the observations at each sensor.

make a single observation and will transmit this observation to the FC. Then, sensors will make independent and identically distributed (iid) observations under $H_{0}$ and none of the sensors can estimate the signal level under $H_{1}$. Consequently, there is no information at the sensors in order to use different quantization thresholds under $H_{1}$. So, it is reasonable to use identical quantization thresholds at each sensor irrespective of their distance to the event location since it cannot be estimated. With constant quantization thresholds, one can estimate the entropy of the observations at the FC. An intuitive idea to have an optimum performance at the $\mathrm{FC}$ is to maximize the entropy of the collected observations under both hypotheses which we call as Maximum Average Entropy (MAE) method. So, we propose to determine the quantization intervals at the sensors as resulting in maximum average entropy under both hypotheses. The entropy of a quantized sensor output can be calculated based on the partitioning of the pdf of the observations at the sensor as shown in Figure 2. In this figure, the number of quantization intervals $M=4$. For a general number of $M$ quantization intervals, there will be $M-1$ thresholds, $\left\{\lambda_{1}, \lambda_{2}, \ldots, \lambda_{M-1}\right\}$, and $M$ partitions with corresponding probabilities $\left\{p_{1}^{H_{i}}, p_{2}^{H_{i}}, \ldots, p_{M}^{H_{i}}\right\}$. Under $H_{0}$, all of the $K$ sensors have iid observations. For a fixed identical threshold set at the sensors, the entropy at the FC under $H_{0}$ can be computed using the closed form formula

$$
F_{H_{0}}=K F_{M}\left(\mathbf{p}_{M}^{H_{0}}\right) \text {. }
$$

where $F_{M}(\cdot)$ denotes the entropy function for the $M$ probability masses corresponding to quantization intervals and $\mathbf{p}_{M}^{H_{0}}=\left[p_{1}^{H_{0}}, p_{2}^{H_{0}}, \ldots, p_{M}^{H_{0}}\right]$ denotes the vector of these probability masses, i.e. the probabilities of the partitions. The resulting entropy at the $\mathrm{FC}$ with the fixed thresholds under $H_{1}$ can be estimated as

$$
\hat{F}_{H_{1}}=\hat{E}\left(-\sum_{k=1}^{K} \sum_{i=1}^{M} p_{k i}^{H_{1}} \log _{2}\left(p_{k i}^{H_{1}}\right)\right) \text { bit }
$$

where $p_{k i}^{H_{1}}$ is the probability mass of the $i$ 'th quantization partition of the $k$ 'th sensor. The expectation, $\hat{E}(\cdot)$, is with 


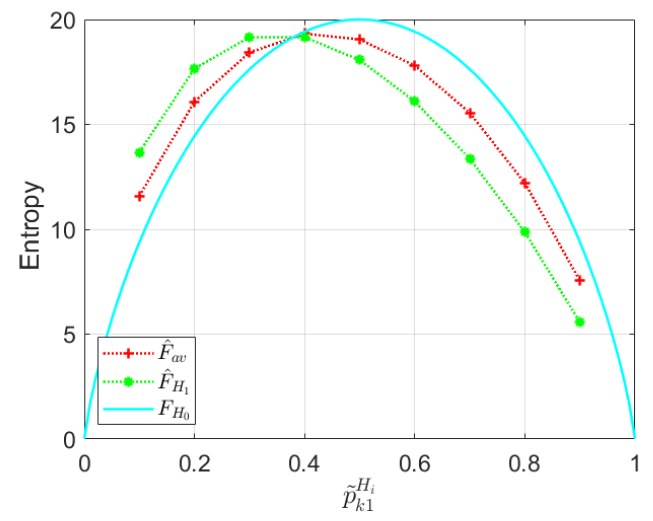

Fig. 3: The entropy functions $F_{H_{0}}, \hat{F}_{H_{1}}$ and $\hat{F}_{\text {av }}$ for binary quantization.

respect to the distribution of $K$ sensors in the sensing range of the sensors defined by a circle within a radius of $r_{\max }$ from the event location. In practice, an estimate of this expectation is performed by averaging the total information of the $K$ sensor locations and AWGN realizations which is called a histogram method [12]. Figure 3 shows the entropy function $F_{H_{0}}, \hat{F}_{H_{1}}$ and $\hat{F}_{\mathrm{av}}=\frac{1}{2}\left(F_{H_{0}}+\hat{F}_{H_{1}}\right)$ for binary quantization. For $M$-ary quantization, $\boldsymbol{\lambda}_{M}=\left[\lambda_{1}, \lambda_{2}, \ldots, \lambda_{M-1}\right]$ denotes the vector of quantization thresholds, the optimal value of which, $\tilde{\lambda}_{M}$, is found as

$$
\tilde{\boldsymbol{\lambda}}_{M}=\arg \max _{\boldsymbol{\lambda}_{M}} \hat{F}_{\mathrm{av}} .
$$

Optimal quantization thresholds correspond to optimal probability mass vectors. Under $H_{1}$, different pdfs are observed at each sensor since the signal level is a function of the distance from the event location. However, under $H_{0}$ the observations at each sensor has the same pdf. So, under $H_{0}$, the optimal probability mass $(\mathrm{pm})$ vector is the same for every sensor which can be given as $\tilde{\mathbf{p}}_{2}^{H_{0}}=\left[\begin{array}{cc}\tilde{p}_{2}^{H_{0}} & 1-\tilde{p}_{2}^{H_{0}}\end{array}\right]$ for binary quantization. This optimal pm vector is $\tilde{\mathbf{p}}_{2}^{H_{0}} \approx\left[\begin{array}{ll}0.4 & 0.6\end{array}\right]$ corresponding to the maximum of $\hat{F}_{\mathrm{av}}$ in Figure 3. In a similar way, we can estimate the optimal vector for 3-level quantization to be $\tilde{\mathbf{p}}_{3}^{H_{0}} \approx\left[\begin{array}{lll}0.27 & 0.32 & 0.4\end{array}\right]$ as shown in Figure 4. Similarly, the optimum pm vector is $\tilde{\mathbf{p}}_{4}^{H_{0}} \approx\left[\begin{array}{llll}0.14 & 0.28 & 0.24 & 0.34\end{array}\right]$ in the case of 4-level quantization.

We can see from Figure 3 that the maximum value of the estimated entropy under $H_{1}$ is $\hat{F}_{H_{1}}=19.1$ bit with a less value than the entropy under $H_{0}$ which is $F_{H_{0}}=20$ bit, which follows Jensen's inequality.

\section{EXPERIMENTAL STUDY}

Monte Carlo simulations have been performed in order to evaluate the detection performance for the proposed method at $\mathrm{SNR}=0 \mathrm{~dB}$ for $K=20$ transmitting sensors and $L=10$. First we have performed simulations using the direct data transmissions (DDT) method, that is assuming the sensor



Fig. 4: The entropy functions $F_{H_{0}}, \hat{F}_{H_{1}}$ and $\hat{F}_{\text {av }}$ for three level quantization.

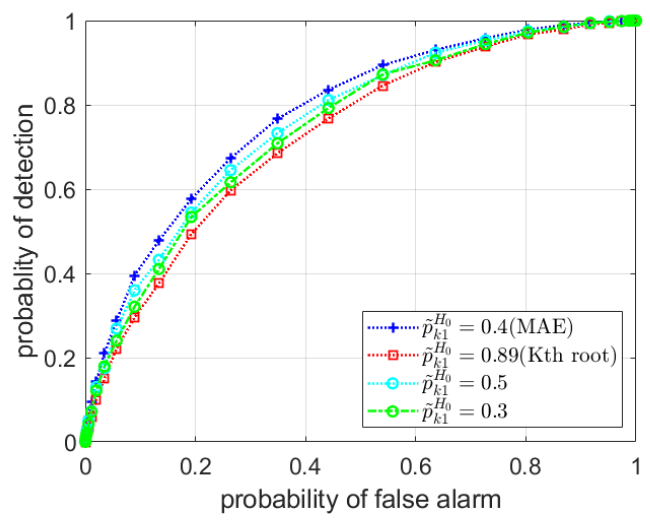

Fig. 5: Comparison between the ROC curves obtained using $\tilde{p}_{2}^{H_{0}}=0.4(\mathrm{MAE}), \tilde{p}_{2}^{H_{0}}=0.89\left(\right.$ Kth root method), $\tilde{p}_{2}^{H_{0}}=0.3$ and $\tilde{p}_{2}^{H_{0}}=0.5$.

outputs arrive error-free to the FC. In Figure 5, the Receiver Operating Characteristics (ROC) curve is calculated by using the optimal quantization intervals from MAE method for the binary data transmission. The optimal threshold is determined as the one resulting in the MAE which corresponds to the local probability of false alarm $\tilde{p}_{2}^{H_{0}}=0.4$. The $K$ 'th root quantization, which uses the $K$ 'th root of the global probability of false alarm $p_{\mathrm{fa}}=0.1$ to find $\mathrm{pmf} \tilde{p}_{2}^{H_{0}}=0.89$ at each sensor, is also provided for the comparison with the proposed method, MAE. K'th root method corresponds to setting the false alarm threshold at the FC to a single "one" coming from any of $\mathrm{K}$ sensors. Additionally, we used two other local $p_{\mathrm{fa}}$ values in order to show that the MAE method outperforms other methods of setting the partitioning threshold. Indeed, this was the case as seen in Figure 5.

The simulation performances for the ternary and quarternary quantizations by using the MAE method are also obtained. For the ternary quantized data transmissions the $\mathrm{MAE}=31.3$ bit which corresponds to $\tilde{\mathbf{p}}_{3}^{H_{0}} \approx\left[\begin{array}{lll}0.44 & 0.33 & 0.23\end{array}\right]$, whereas the $\mathrm{MAE}=38.7$ bit for quarternary data transmissions at $\tilde{\mathbf{p}}_{4}^{H_{0}} \approx$ $\left[\begin{array}{llll}0.14 & 0.28 & 0.24 & 0.34\end{array}\right]$. 




Fig. 6: ROC curves of data with different levels (2,3 and 4) of quantization and non-quantized data.

ROC curves obtained with different levels of quantization using MAE method and non-quantized data are shown, in Figure 6. This figure depicts that at global false alarm probability $p_{\mathrm{fa}}=0.2$ probability of detection $p_{d}=0.79,0.72,0.63$ and 0.58 for the Gaussian, quarternary, ternary, and binary data transmissions, respectively. Next, we consider a simplified realistic channel model for transmission of sensor data to the FC, namely binary symmetric channel (BSC). In this model, a bit transmitted from sensor $k$ arrives at the FC without error with probability $\left(1-b_{k}\right)$ and correspondingly inverted with probability $b_{k}$. In Figure 7, the ROC curves of binary sensor data transmission obtained by using the MAE method are plotted for the case of DDT and BSC. For bit error rate $b_{k}=0.1$ and $p_{\mathrm{fa}}=0.4$, the values of the $p_{\mathrm{d}}$ as 0.80 and 0.72 are obtained for DDT and BSC, respectively. In a similar way, a comparison between the DDT and noisy channel data transmission for the same total probability of error in the channel have been performed for the ternary and quarternary quantized and non-quantized, i.e. Gaussian data transmissions and it shows that the loss in the probability of detection between the DDT and the noisy channel data transmissions at specific probability of false alarm decreases by increasing the number of transmitted bits to the FC.

\section{CONCLUSION}

This paper proposed a multiple level quantization method for the sensor outputs in a WSN composed of uniformly deployed sensors and a FC used to detect a static event which can be observed as signals emitted from a point source. The developed method is a NP criterion based distributed detection scheme depending on MAE. Obtained ROCs show that the MAE method for quantizing sensor outputs performs significantly better than the K'th root method or other choices for the thresholds in binary quantization of sensor outputs. The performance obtained using MAE method has been also investigated for ternary and quarternary quantization. Increasing number of levels in quantization have resulted in better performance as expexted, however more bits in transmission

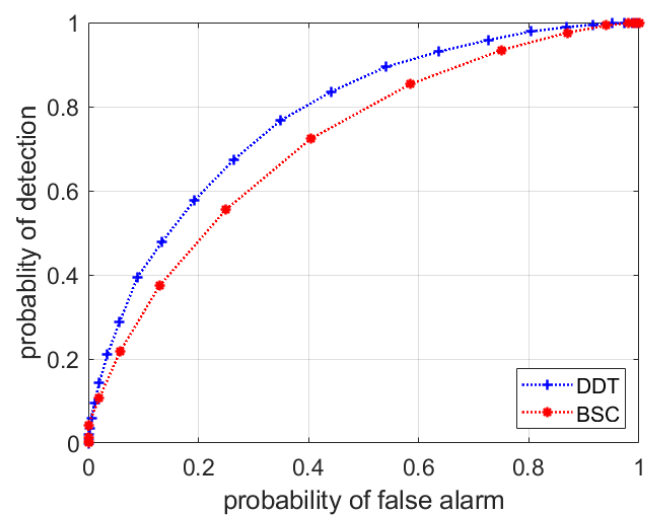

Fig. 7: ROC curves with fusion of binary sensor data obtained using either error-free or binary symmetric channels.

are needed for a performance approaching the one of nonquantized data transmission to the FC.

The effects of the channels from the sensors to the FC have been also investigated using simple channel models like the BSC in the case of binary quantization. The loss in probability of detection by including the channel is observed to decrease when the level of quantization is increased for 10 percent probability of error introduced by the simplified symmetric channel and for the global false alarm rate 0.1 at the FC.

\section{REFERENCES}

[1] J. N. Tsitsiklis, "Decentralized detection," Advances in Statistical Signal Processing, vol. 2, no. 2, pp. 297-344, 1993.

[2] M. A. Al-Jarrah, A. Al-Dweik, "Decision Fusion in Distributed Cooperative Wireless Sensor Networks," IEEE Transaction on Vehicular Tech., vol.68, no. 1, pp. 797-811, Jan. 2019.

[3] D. Ciuonzo, G. Romano, and P. Salvo Rossi, "Optimality of received energy in decision fusion over Rayleigh fading diversity MAC with nonidentical sensors," IEEE Transaction on Signal Process., vol. 61, no. 1, pp. 22-27, Jan. 2013.

[4] Stella I Johnsi, S. Radha, "Investigations on distributed detection performance of Neyman-Pearson detection scheme using constructive interference technique for WSN," International Conference on Wireless Communication and Sensor Computing, 2010.

[5] R. R. Tenney and N. R. Sandell, "Detection with distributed sensors," IEEE Transaction on Aerospace Electronics System, vol. 17, no. 4, pp. 501-510, Jul. 1981.

[6] Z. Chair and P. K. Varshney, "Optimal data fusion in multiple sensor detection systems," IEEE Transaction on Aerospace Electronics System, vol. 22, no. 1, pp. 98-101, Jan. 1986.

[7] S. C. A. Thomopoulos, R. Viswanathan, and D. C. Bougoulias, "Optimal decision fusion in multiple sensor systems," IEEE Transaction on Aerospace Electronics System, vol. 23, no. 5, pp. 644-653, Sept. 1987.

[8] M. Al-Ibrahim and S. AlHakeem, "Optimization of a serial distributed detection system with 2 bits communication constraint," Int. J. Syst. Sci.,vol. 32, no. 9, pp. 1169-1175, 2001.

[9] C. C. Lee and J. J. Chao, "Optimum local decision space partitioning for distributed detection," IEEE Transaction on Aerospace Electronics System, AES,vol. 25, no. 7, pp. 536-544, Jul. 1989.

[10] C. Altay and H. Delic, "Optimal quantization intervals in distributed detection," IEEE Transaction on Aerospace Electronics System, vol. 52, no. 1, pp. 38-48, February 2016.

[11] S. M. Kay, Fundamentals of Statistical Signal Processing, Volume 2: Detection Theory. Prentice Hall PTR, Jan. 1998.

[12] Bercher J.-F., Vignat C. "Estimating the entropy of a signal with applications," IEEE Transaction on Signal Processing, 48, (6), pp. 16871694, 2000. 